\title{
Analysis of the M/G/1 retrial queueing model with server breakdowns
}

\author{
Geni Gupur
}

Received: 25 March 2010 / Revised: 29 May 2010 / Accepted: 23 June 2010 /

Published online: 21 July 2010

(C) The Author(s) 2010. This article is published with open access at Springerlink.com

\begin{abstract}
By using the Hille-Yosida theorem and the Phillips theorem in functional analysis we prove that the $\mathrm{M} / \mathrm{G} / 1$ retrial queueing model with server breakdowns has a unique nonnegative time-dependent solution. Next, when the service completion rate is a constant, by studying spectral properties of the operator corresponding to the model we study asymptotic behavior of its time-dependent solution. First of all, through considering the resolvent set of the adjoint operator of the operator we obtain that all points on the imaginary axis except zero belong to the resolvent set of the operator. In addition, we prove that zero is not an eigenvalue of the operator. Our results show that the time-dependent solution of the model strongly converges to zero.
\end{abstract}

Keywords The M/G/1 retrial queueing model with server breakdowns · $C_{0}$-semigroup $\cdot$ Dispersive operator $\cdot$ Resolvent set $\cdot$ Eigenvalue

Mathematics Subject Classification (2000) Primary 47D03 - 47A10;

Secondary $60 \mathrm{~K} 25$

\section{Introduction}

Retrial queueing systems have been used to model many problems in telephone switching systems, telecommunication networks, computer networks, computer and communication systems. The first result on M/G/1 retrial queues is due to Keilson et al.

This work was supported by the Natural Science Foundation of China (No: 10861011).

G. Gupur ( $\varangle)$

College of Mathematics and Systems Science, Xinjiang University,

Urumqi 830046, People's Republic of China

e-mail: genigupur@yahoo.cn 
[8] who used the method of supplementary variables. They obtained the joint distribution of the channel state and the queueing length in steady state. Later, Aleksandrov [2] and Falin [5] independently considered the case of general service times using different methods. Some of its variations have been studied by a number of authors, see Kulkarni [9], Choi and Park [4], Yang and Templeton [12], for instance. In 2001, Wang et al. [11] studied the M/G/1 retrial queue with server breakdowns in which the failure states of the server are absorbing states. By using the supplementary variable technique they established the corresponding queueing model and obtained explicit expressions of some reliability indices such as the availability, failure frequency for steady-state cases under the following hypothesis: "The time-dependent solution of the model converges to a nonzero steady-state solution." By reading their paper we find that the above hypothesis, in fact, implies the following two hypotheses:

Hypothesis 1 The model has a nonnegative time-dependent solution.

Hypothesis 2 The time-dependent solution of the model converges to a nonzero steady-state solution.

So far any other results about this model have not been found in the literature. In this paper, we do dynamic analysis for the queueing model, that is, we study the above two hypotheses. First of all, by using the Hille-Yosida theorem and the Phillips theorem we prove that the model has a unique nonnegative time-dependent solution and therefore we obtain that the Hypothesis 1 holds. Next, when the service completion rate is a constant, we study the asymptotic behavior of its time-dependent solution, i.e., we study the Hypothesis 2. When the service completion rate is a constant, the $\mathrm{M} / \mathrm{G} / 1$ retrial queueing model with server breakdowns is called the $\mathrm{M} / \mathrm{M} / 1$ retrial queueing model with server breakdowns. Firstly, we determine the resolvent set of the adjoint operator of the operator corresponding to the $\mathrm{M} / \mathrm{M} / 1$ retrial queueing model with server breakdowns, then we prove that 0 is not an eigenvalue of the operator, which shows that the steady-state solution of the model is equal to zero. Hence, it is impossible that the time-dependent solution of the model converges to a nonzero steady-state solution. In other words, the Hypothesis 2 does not hold. If we can verify that 0 is not in the residue spectrum of the operator, then from the above results we conclude that the time-dependent solution of the model strongly converges to zero. That is to say, the results in Wang et al. [11] do not hold.

According to Wang et al. [11], the M/G/1 retrial queueing system with server breakdowns can be described by the following system of equations:

$$
\begin{aligned}
\frac{d p_{I, i, 0}(t)}{d t}=-(\lambda+i \theta) p_{I, i, 0}(t) & +\int_{0}^{\infty} \mu(x) p_{w, i, 1}(x, t) d x, i \geq 0 \\
\frac{\partial p_{w, 0,1}(x, t)}{\partial t}+\frac{\partial p_{w, 0,1}(x, t)}{\partial x}= & -(\lambda+\alpha+\mu(x)) p_{w, 0,1}(x, t), \\
\frac{\partial p_{w, i, 1}(x, t)}{\partial t}+\frac{\partial p_{w, i, 1}(x, t)}{\partial x}= & -(\lambda+\alpha+\mu(x)) p_{w, i, 1}(x, t) \\
& +\lambda p_{w, i-1,1}(x, t), \quad i \geq 1
\end{aligned}
$$




$$
\begin{aligned}
& p_{w, i, 1}(0, t)=\lambda p_{I, i, 0}(t)+(i+1) \theta p_{I, i+1,0}(t), \quad i \geq 0 \\
& p_{I, 0,0}(0)=1, \quad p_{I, i, 0}(0)=0, \quad i \geq 1 ; \quad p_{w, j, 1}(x, 0)=0, \quad j \geq 0 .
\end{aligned}
$$

Here $(x, t) \in[0, \infty) \times[0, \infty) ; p_{I, i, 0}(t)(i \geq 0)$ is the probability that the server is idle and there are $i$ customers in the retrial group at time $t ; p_{w, i, 1}(x, t)(i \geq 0)$ is the joint probability that at time $t$ there are $i$ customers in the retrial group and the server is up and the customer is being served with elapsed service time $x ; \lambda$ is the arrival rate of customers; $\alpha$ is the server failing rate; $\mu(x)$ is the service completion rate, $\theta$ is the successive inter-retrial times of customers.

For convenience, we introduce a notation as follows.

$$
\Gamma=\left(\begin{array}{cccccc}
\lambda & \theta & 0 & 0 & 0 & \cdots \\
0 & \lambda & 2 \theta & 0 & 0 & \cdots \\
0 & 0 & \lambda & 3 \theta & 0 & \cdots \\
0 & 0 & 0 & \lambda & 4 \theta & \cdots \\
\vdots & \vdots & \vdots & \vdots & \vdots & \vdots
\end{array}\right)
$$

Choose a state space as follows.

$$
X=\left\{\begin{array}{l|l}
\left(p_{I}, p_{w}\right) & \begin{array}{l}
p_{I}=\left(p_{I, 0,0}, p_{I, 1,0}, p_{I, 2,0}, \ldots\right) \in l^{1} \\
p_{w}=\left(p_{w, 0,1}, p_{w, 1,1}, p_{w, 2,1}, \ldots\right) \\
\in L^{1}[0, \infty) \times L^{1}[0, \infty) \times L^{1}[0, \infty) \times \cdots \\
\left\|\left(p_{I}, p_{w}\right)\right\|=\sum_{i=0}^{\infty}\left|p_{I, i, 0}\right|+\sum_{i=0}^{\infty}\left\|p_{w, i, 1}\right\|_{L^{1}[0, \infty)}<\infty
\end{array}
\end{array}\right\}
$$

It is obvious that $X$ is a Banach space and also a Banach lattice. In the following we define operators and their domains.

$$
\begin{aligned}
& A\left(p_{I}, p_{w}\right)=\left(\left(\begin{array}{ccccc}
-\lambda & 0 & 0 & 0 & \cdots \\
0 & -(\lambda+\theta) & 0 & 0 & \ldots \\
0 & 0 & -(\lambda+2 \theta) & 0 & \ldots \\
0 & 0 & 0 & -(\lambda+3 \theta) & \ldots \\
\vdots & \vdots & \vdots & \vdots & \vdots
\end{array}\right)\left(\begin{array}{c}
p_{I, 0,0} \\
p_{I, 1,0} \\
p_{I, 2,0} \\
p_{I, 3,0} \\
\vdots
\end{array}\right)\right. \\
& \left.\left(\begin{array}{ccccc}
-\frac{d}{d x} & 0 & 0 & 0 & \cdots \\
0 & -\frac{d}{d x} & 0 & 0 & \cdots \\
0 & 0 & -\frac{d}{d x} & 0 & \cdots \\
0 & 0 & 0 & -\frac{d}{d x} & \cdots \\
\vdots & \vdots & \vdots & \vdots & \vdots
\end{array}\right)\left(\begin{array}{c}
p_{w, 0,1} \\
p_{w, 1,1} \\
p_{w, 2,1} \\
p_{w, 3,1} \\
\vdots
\end{array}\right)\right) \\
& D(A)=\left\{\begin{array}{l|l}
\left(p_{I}, p_{w}\right) \in X & \begin{array}{l}
p_{w, i, 1}(i \geq 0) \text { are absolutely continuous } \\
\text { functions and satisfy } p_{w}(0)=\Gamma p_{I}, \\
\sum_{i=0}^{\infty}\left\|\frac{d p_{w, i, 1}}{d x}\right\|_{L^{1}[0, \infty)}<\infty
\end{array}
\end{array}\right\},
\end{aligned}
$$




$$
\begin{aligned}
U\left(p_{I}, p_{w}\right)= & \left(\left(\begin{array}{cccc}
0 & 0 & 0 & \cdots \\
0 & 0 & 0 & \cdots \\
0 & 0 & 0 & \cdots \\
\vdots & \vdots & \vdots & \vdots
\end{array}\right)\left(\begin{array}{c}
p_{I, 0,0} \\
p_{I, 1,0} \\
p_{I, 2,0} \\
\vdots
\end{array}\right),\right. \\
& \left.\left(\begin{array}{ccccc}
\omega & 0 & 0 & 0 & \cdots \\
\lambda & \omega & 0 & 0 & \cdots \\
0 & \lambda & \omega & 0 & \cdots \\
\vdots & \vdots & \vdots & \vdots & \vdots
\end{array}\right)\left(\begin{array}{c}
p_{w, 0,1} \\
p_{w, 1,1} \\
p_{w, 2,1} \\
\vdots
\end{array}\right)\right), \quad D(U)=X,
\end{aligned}
$$

where $\omega=-(\lambda+\alpha+\mu(x))$.

$$
E\left(p_{I}, p_{w}\right)=\left(\left(\begin{array}{l}
\int_{0}^{\infty} \mu(x) p_{w, 0,1}(x) d x \\
\int_{0}^{\infty} \mu(x) p_{w, 1,1}(x) d x \\
\int_{0}^{\infty} \mu(x) p_{w, 2,1}(x) d x \\
\vdots \\
\vdots \\
0
\end{array}\right), \quad\left(\begin{array}{c}
0 \\
0 \\
0 \\
\end{array}\right), \quad D(E)=X .\right.
$$

Then the above system of Eqs. (1.1)-(1.5) can be rewritten as an abstract Cauchy problem:

$$
\left\{\begin{array}{l}
\frac{d\left(p_{I}, p_{w}\right)(t)}{d t}=(A+U+E)\left(p_{I}, p_{w}\right)(t), \quad t \in(0, \infty) \\
\left(p_{I}, p_{w}\right)(0)=\left(\left(\begin{array}{c}
1 \\
0 \\
\vdots
\end{array}\right),\left(\begin{array}{c}
0 \\
0 \\
\vdots
\end{array}\right)\right)
\end{array}\right.
$$

\section{Well-posedness of the system (1.6)}

Theorem 2.1 If $\mu(x)$ satisfies $\mu=\sup _{x \in[0, \infty)} \mu(x)<\infty$, then $A+U+E$ generates a positive contraction $C_{0}$-semigroup $T(t)$.

Proof We split the proof of this theorem into 4 steps. First of all, we estimate $\|(\gamma I-$ $A)^{-1} \|$, next we verify that $D(A)$ is dense in $X$. Thirdly, we prove that $U$ and $E$ are bounded linear operators. Lastly, we check that $A+U+E$ is dispersive and therefore we obtain the desired result. 
For any given $\left(y_{I}, y_{w}\right) \in X$, consider the equation $(\gamma I-A)\left(p_{I}, p_{w}\right)=\left(y_{I}, y_{w}\right)$, that is,

$$
\begin{gathered}
(\gamma+\lambda+i \theta) p_{I, i, 0}=y_{I, i, 0}, \quad i \geq 0, \\
\frac{d p_{w, i, 1}(x)}{d x}=-\gamma p_{w, i, 1}(x)+y_{w, i, 1}(x), \quad i \geq 0 \\
p_{w, i, 1}(0)=\lambda p_{I, i, 0}+(i+1) \theta p_{I, i+1,0}, \quad i \geq 0 .
\end{gathered}
$$

By solving (2.1) and (2.2), we have

$$
\begin{gathered}
p_{I, i, 0}=\frac{1}{\gamma+\lambda+i \theta} y_{I, i, 0}, \quad i \geq 0, \\
p_{w, i, 1}(x)=a_{i} e^{-\gamma x}+e^{-\gamma x} \int_{0}^{x} y_{w, i, 1}(\tau) e^{\gamma \tau} d \tau, \quad i \geq 0 .
\end{gathered}
$$

By combining (2.5) with (2.3) and (2.4), it follows that

$$
\begin{aligned}
a_{i} & =p_{w, i, 1}(0)=\lambda p_{I, i, 0}+(i+1) \theta p_{I, i+1,0} \\
& =\frac{\lambda}{\gamma+\lambda+i \theta} y_{I, i, 0}+\frac{(i+1) \theta}{\gamma+\lambda+(i+1) \theta} y_{I, i+1,0}, \quad i \geq 0 .
\end{aligned}
$$

Through inserting (2.6) into (2.5) and using the Fubini theorem, we deduce, without loss of generality assuming $\gamma>0$,

$$
\begin{aligned}
\left\|p_{w, i, 1}\right\|_{L^{1}[0, \infty)} \leq & \int_{0}^{\infty}\left|a_{i}\right| e^{-\gamma x} d x+\int_{0}^{\infty} e^{-\gamma x} \int_{0}^{x}\left|y_{w, i, 1}(\tau)\right| e^{\gamma \tau} d \tau d x \\
= & \frac{1}{\gamma}\left|a_{i}\right|+\int_{0}^{\infty}\left|y_{w, i, 1}(\tau)\right| e^{\gamma \tau} \int_{\tau}^{\infty} e^{-\gamma x} d x d \tau \\
= & \frac{1}{\gamma}\left|a_{i}\right|+\frac{1}{\gamma}\left\|y_{w, i, 1}\right\|_{L^{1}[0, \infty)}\left[\frac{\lambda}{\gamma+\lambda+i \theta}\left|y_{I, i, 0}\right|+\frac{(i+1) \theta}{\gamma+\lambda+(i+1) \theta}\left|y_{I, i+1,0}\right|\right\} \\
\leq & \frac{1}{\gamma}\left\{\frac{1}{\gamma+y_{w, i, 1} \|_{L^{1}[0, \infty)}}\right. \\
& +\frac{1}{\gamma}\left\|y_{w, i, 1}\right\|_{L^{1}[0, \infty)}+\frac{\lambda(\gamma+\lambda+i \theta)}{\gamma\left(\gamma+y_{I, i, 0} \mid\right.} \\
& +\frac{(i+1) \theta}{\gamma[\gamma+\lambda+(i+1) \theta]}\left|y_{I, i+1,0}\right|, \quad i \geq 0 .
\end{aligned}
$$


From (2.4) and (2.7), we estimate, noting $\gamma>0$,

$$
\begin{aligned}
\left\|\left(p_{I}, p_{w}\right)\right\|= & \sum_{i=0}^{\infty}\left|p_{I, i, 0}\right|+\sum_{i=0}^{\infty}\left\|p_{w, i, 1}\right\|_{L^{1}[0, \infty)} \\
\leq & \sum_{i=0}^{\infty} \frac{1}{\gamma+\lambda+i \theta}\left|y_{I, i, 0}\right|+\sum_{i=0}^{\infty} \frac{1}{\gamma}\left\|y_{w, i, 1}\right\|_{L^{1}[0, \infty)} \\
& +\sum_{i=0}^{\infty} \frac{\lambda}{\gamma(\gamma+\lambda+i \theta)}\left|y_{I, i, 0}\right|+\sum_{i=0}^{\infty} \frac{(i+1) \theta}{\gamma[\gamma+\lambda+(i+1) \theta]}\left|y_{I, i+1,0}\right| \\
\leq & \sum_{i=0}^{\infty}\left[\frac{1}{\gamma+\lambda+i \theta}+\frac{\lambda}{\gamma(\gamma+\lambda+i \theta)}+\frac{(i+1) \theta}{\gamma[\gamma+\lambda+(i+1) \theta]}\right]\left|y_{I, i, 0}\right| \\
& +\frac{1}{\gamma} \sum_{i=0}^{\infty}\left\|y_{w, i, 1}\right\|_{L^{1}[0, \infty)} .
\end{aligned}
$$

To simplify (2.8), we discuss the properties of the following function.

$$
f(x)=\frac{1}{\gamma+\lambda+x}+\frac{\lambda}{\gamma(\gamma+\lambda+x)}+\frac{x+\theta}{\gamma(\gamma+\lambda+x+\theta)}, \quad x \in[0, \infty) .
$$

It is easy to see that $f(x)$ is differentiable on $[0, \infty)$. Moreover,

$$
\begin{aligned}
\frac{d f(x)}{d x} & =-\frac{1}{(\gamma+\lambda+x)^{2}}-\frac{\lambda}{\gamma(\gamma+\lambda+x)^{2}}+\frac{1}{\gamma} \frac{\gamma+\lambda}{(\gamma+\lambda+x+\theta)^{2}} \\
& =-\frac{\gamma+\lambda}{\gamma(\gamma+\lambda+x)^{2}}+\frac{\gamma+\lambda}{\gamma(\gamma+\lambda+x+\theta)^{2}} \\
& =\frac{\gamma+\lambda}{\gamma}\left[\frac{1}{(\gamma+\lambda+x+\theta)^{2}}-\frac{1}{(\gamma+\lambda+x)^{2}}\right]<0 .
\end{aligned}
$$

This shows that $f(x)$ is strictly monotone decreasing. Therefore $f(x)<f(0), \quad x \in$ $(0, \infty)$, i.e.,

$$
f(x)<f(0)=\frac{1}{\gamma+\lambda}+\frac{\lambda}{\gamma(\gamma+\lambda)}+\frac{\theta}{\gamma(\gamma+\lambda+\theta)} .
$$

By using this formula, we simplify (2.8) as follows when $\gamma>\lambda+\theta$.

$$
\begin{aligned}
\left\|\left(p_{I}, p_{w}\right)\right\| \leq & \sum_{i=0}^{\infty}\left[\frac{1}{\gamma+\lambda}+\frac{\lambda}{\gamma(\gamma+\lambda)}+\frac{\theta}{\gamma(\gamma+\lambda+\theta)}\right]\left|y_{I, i, 0}\right| \\
& +\frac{1}{\gamma} \sum_{i=0}^{\infty}\left\|y_{w, i, 1}\right\|_{L^{1}[0, \infty)}
\end{aligned}
$$




$$
\begin{aligned}
= & \sum_{i=0}^{\infty}\left[\frac{\gamma+\lambda}{\gamma(\gamma+\lambda)}+\frac{\theta}{\gamma(\gamma+\lambda+\theta)}\right]\left|y_{I, i, 0}\right| \\
& +\frac{1}{\gamma} \sum_{i=0}^{\infty}\left\|y_{w, i, 1}\right\|_{L^{1}[0, \infty)} \\
= & \sum_{i=0}^{\infty}\left[\frac{1}{\gamma}+\frac{\theta}{\gamma(\gamma+\lambda+\theta)}\right]\left|y_{I, i, 0}\right| \\
& +\frac{1}{\gamma} \sum_{i=0}^{\infty}\left\|y_{w, i, 1}\right\|_{L^{1}[0, \infty)} \\
= & \frac{\gamma+\lambda+2 \theta}{\gamma(\gamma+\lambda+\theta)} \sum_{i=0}^{\infty}\left|y_{I, i, 0}\right|+\frac{1}{\gamma} \sum_{i=0}^{\infty}\left\|y_{w, i, 1}\right\|_{L^{1}[0, \infty)} \\
\leq & \frac{1}{\gamma-\lambda-\theta} \sum_{i=0}^{\infty}\left|y_{I, i, 0}\right|+\frac{1}{\gamma-\lambda-\theta} \sum_{i=0}^{\infty}\left\|y_{w, i, 1}\right\|_{L^{1}[0, \infty)} \\
= & \frac{1}{\gamma-\lambda-\theta}\left\|\left(y_{I}, y_{w}\right)\right\| .
\end{aligned}
$$

In (2.9), we have applied the following inequality.

$$
\begin{aligned}
\lambda & (\gamma+\lambda+2 \theta)+\theta(\lambda+2 \theta)>0 \\
& \Longleftrightarrow \gamma \theta+\lambda(\gamma+\lambda+2 \theta)+\theta(\lambda+2 \theta)>\gamma \theta \\
& \Longleftrightarrow \lambda(\gamma+\lambda+2 \theta)+\theta(\gamma+\lambda+2 \theta)>\gamma \theta \\
& \Longleftrightarrow \gamma(\gamma+\lambda+\theta)+\lambda(\gamma+\lambda+2 \theta)+\theta(\gamma+\lambda+2 \theta)>\gamma \theta+\gamma(\gamma+\lambda+\theta) \\
& \Longleftrightarrow \gamma(\gamma+\lambda+\theta)+(\theta+\lambda)(\gamma+\lambda+2 \theta)>\gamma(\gamma+\lambda+2 \theta) \\
& \Longleftrightarrow \gamma(\gamma+\lambda+\theta)>\gamma(\gamma+\lambda+2 \theta)-(\theta+\lambda)(\gamma+\lambda+2 \theta) \\
& \Longleftrightarrow \gamma(\gamma+\lambda+\theta)>(\gamma-\theta-\lambda)(\gamma+\lambda+2 \theta) \\
& \Longleftrightarrow \frac{\gamma+\lambda+2 \theta}{\gamma(\gamma+\lambda+\theta)}<\frac{1}{\gamma-\lambda-\theta} .
\end{aligned}
$$

By (2.9),

$$
(\gamma I-A)^{-1}: X \rightarrow D(A), \quad\left\|(\gamma I-A)^{-1}\right\| \leq \frac{1}{\gamma-\lambda-\theta}, \quad \forall \gamma>\lambda+\theta
$$

Secondly, we will prove that $D(A)$ is dense in $X$. Since $\left(p_{I}, p_{w}\right) \in X$ implies that $\forall \epsilon>0, \exists N, \forall n>N$ such that $\sum_{i=N+1}^{\infty}\left|p_{I, i, 0}\right|<\epsilon, \sum_{i=N+1}^{\infty}\left\|p_{w, i, 1}\right\|_{L^{1}[0, \infty)}<\epsilon$, 
the set

$$
W=\left\{\begin{array}{l|l}
\left(p_{I}^{0}, p_{w}^{0}\right) & \begin{array}{l}
p_{I}^{0}=\left(p_{I, 0,0}, p_{I, 1,0}, \ldots, p_{I, N, 0}, 0,0, \ldots\right), \\
p_{w}^{0}(x)=\left(p_{w, 0,1}(x), p_{w, 1,1}(x), \ldots, p_{w, N, 1}(x), 0,0, \ldots\right), \\
p_{I, i, 0} \in \mathbb{R}, p_{w, i, 1} \in L^{1}[0, \infty), i=0,1, \ldots, N \\
N \text { is a finite positive integer }
\end{array}
\end{array}\right\}
$$

is dense in $X$. By Adams [1], we know that the set

$$
Q=\left\{\begin{array}{l|l}
\left(p_{I}^{*}, p_{w}^{*}\right) & \begin{array}{l}
p_{I}^{*}=\left(p_{I, 0,0}, p_{I, 1,0}, \ldots, p_{I, K, 0}, 0,0, \ldots\right) \\
p_{w}^{*}(x)=\left(p_{w, 0,1}(x), p_{w, 1,1}(x), \ldots, p_{w, K, 1}(x), 0,0, \ldots\right), \\
p_{I, i, 0} \in \mathbb{R}, p_{w, i, 1} \in C^{\infty}[0, \infty), \exists C_{i}>0 \\
\text { such that } p_{w, i, 1}(x)=0, \forall x \in\left[0, C_{i}\right], i=0,1, \ldots, N \\
N \text { is a finite positive integer }
\end{array}
\end{array}\right\}
$$

is dense in $W$. Hence, in order to prove denseness of $D(A)$, it suffices to prove that $D(A)$ is dense in $Q$. Take

$$
\begin{aligned}
\left(p_{I}, p_{w}\right) & \in Q, p_{I}=\left(p_{I, 0,0}, p_{I, 1,0}, \ldots, p_{I, K, 0}, 0,0, \ldots\right) \\
p_{w}(x) & =\left(p_{w, 0,1}(x), p_{w, 1,1}(x), \ldots, p_{w, K, 1}(x), 0,0, \ldots\right), \\
p_{w, i, 1}(x) & =0, x \in\left[0, C_{i}\right], i=0,1, \ldots, K
\end{aligned}
$$

then $p_{w, i, 1}(x)=0$ for $x \in[0, s], i=0,1, \ldots, K$, here $0<s=\min _{0 \leq i \leq K} C_{i}$. We define

$$
\begin{aligned}
f_{I} & =\left(f_{I, 0,0}, f_{I, 1,0}, \ldots, f_{I, K, 0}, 0,0, \ldots\right) \\
& =\left(p_{I, 0,0}, p_{I, 1,0}, \ldots, p_{I, K, 0}, 0,0, \ldots\right), \\
f_{w, i, 1}(0) & =\lambda p_{I, i, 0}+(i+1) \theta p_{I, i+1,0}, \quad i=0,1, \ldots, K-1, \\
f_{w, K, 1}(0) & =\lambda p_{I, K, 0}, \\
f_{w}(x) & =\left(f_{w, 0,1}(x), f_{w, 1,1}(x), \ldots, f_{w, K, 1}(x), 0,0, \ldots\right), \\
f_{w, i, 1}(x) & =\left\{\begin{array}{ll}
f_{w, i, 1}(0)\left(1-\frac{x}{s}\right)^{2} & x \in[0, s) \\
p_{w, i, 1}(x) & x \in[s, \infty)
\end{array}, i=0,1, \ldots, K,\right.
\end{aligned}
$$


then it is easy to check $\left(f_{I}, f_{w}\right) \in D(A)$. Moreover,

$$
\begin{aligned}
\left\|\left(p_{I}, p_{w}\right)-\left(f_{I}, f_{w}\right)\right\| & =\left\|\left(p_{I}-f_{I}, p_{w}-f_{w}\right)\right\| \\
& =\sum_{i=0}^{K} \int_{0}^{\infty}\left|p_{w, i, 1}(x)-f_{w, i, 1}(x)\right| d x \\
& =\sum_{i=0}^{K} \int_{0}^{s}\left|f_{w, i, 1}(0)\right|\left(1-\frac{x}{s}\right)^{2} d x \\
& =\sum_{i=0}^{K}\left|f_{w, i, 1}(0)\right| \frac{s}{3} \rightarrow 0 \text { as } s \rightarrow 0 .
\end{aligned}
$$

This shows that $D(A)$ is dense in $Q$. In other words, $D(A)$ is dense in $X$.

Next we will verify that $U$ and $E$ are bounded linear operators. From the definitions of $U$ and $E$,

$$
\begin{aligned}
U\left(p_{I}, p_{w}\right)(x)= & \left(\left(\begin{array}{l}
0 \\
0 \\
0 \\
\vdots
\end{array}\right),\left(\begin{array}{c}
-(\lambda+\alpha+\mu(x)) p_{w, 0,1}(x) \\
-(\lambda+\alpha+\mu(x)) p_{w, 1,1}(x)+\lambda p_{w, 0,1}(x) \\
-(\lambda+\alpha+\mu(x)) p_{w, 2,1}(x)+\lambda p_{w, 1,1}(x) \\
\vdots
\end{array}\right)\right), \\
E\left(p_{I}, p_{w}\right)(x)= & \left(\left(\begin{array}{c}
\int_{0}^{\infty} \mu(x) p_{w, 0,1}(x) d x \\
0 \\
\int_{0}^{\infty} \mu(x) p_{w, 1,1}(x) d x \\
\int_{0}^{\infty} \mu(x) p_{w, 2,1}(x) d x \\
\vdots \\
0 \\
0 \\
0
\end{array}\right),\right.
\end{aligned}
$$

we have, for any $\left(p_{I}, p_{w}\right) \in X$,

$$
\begin{aligned}
\left\|U\left(p_{I}, p_{w}\right)\right\| \leq & \sum_{i=0}^{\infty} \int_{0}^{\infty}\left|(\lambda+\alpha+\mu(x)) p_{w, i, 1}(x)\right| d x \\
& +\sum_{i=0}^{\infty} \int_{0}^{\infty}\left|\lambda p_{w, i, 1}(x)\right| d x \\
\leq & (\lambda+\alpha+\mu) \sum_{i=0}^{\infty} \int_{0}^{\infty}\left|p_{w, i, 1}(x)\right| d x
\end{aligned}
$$




$$
\begin{aligned}
& +\lambda \sum_{i=0}^{\infty} \int_{0}^{\infty}\left|p_{w, i, 1}(x)\right| d x \\
= & (2 \lambda+\alpha+\mu) \sum_{i=0}^{\infty}\left\|p_{w, i, 1}\right\|_{L^{1}[0, \infty)} \\
\leq & (2 \lambda+\alpha+\mu)\left\|\left(p_{I}, p_{w}\right)\right\| . \\
\left\|E\left(p_{I}, p_{w}\right)\right\| \leq & \sum_{i=0}^{\infty} \int_{0}^{\infty}\left|\mu(x) p_{w, i, 1}(x)\right| d x \\
\leq & \mu \sum_{i=0}^{\infty} \int_{0}^{\infty}\left|p_{w, i, 1}(x)\right| d x \\
= & \mu \sum_{i=0}^{\infty}\left\|p_{w, i, 1}\right\|_{L^{\infty}[0, \infty) \leq \mu\left\|\left(p_{I}, p_{w}\right)\right\| .}
\end{aligned}
$$

(2.10) and (2.11) show that $U$ and $E$ are bounded operators. It is easy to see that $U$ and $E$ are linear. In conclusion, $U$ and $E$ are bounded linear operators.

Lastly, we will prove that $A+U+E$ is a dispersive operator. For $\left(p_{I}, p_{w}\right) \in X$, take $(\phi, \psi)$ as

$$
\begin{aligned}
\phi & =\left(\frac{\left[p_{I, 0,0}\right]^{+}}{p_{I, 0,0}}, \frac{\left[p_{I, 1,0}\right]^{+}}{p_{I, 1,0}}, \frac{\left[p_{I, 2,0}\right]^{+}}{p_{I, 2,0}}, \ldots\right), \\
\psi(x) & =\left(\frac{\left[p_{w, 0,1}(x)\right]^{+}}{p_{w, 0,1}(x)}, \frac{\left[p_{w, 1,1}(x)\right]^{+}}{p_{w, 1,1}(x)}, \frac{\left[p_{w, 2,1}(x)\right]^{+}}{p_{w, 2,1}(x)}, \ldots\right), \\
{\left[p_{I, i, 0}\right]^{+} } & =\left\{\begin{array}{ll}
p_{I, i, 0} & p_{I, i, 0}>0 \\
0 & p_{I, i, 0} \leq 0,
\end{array}, \quad i \geq 0,\right. \\
{\left[p_{w, i, 1}(x)\right]^{+} } & =\left\{\begin{array}{ll}
p_{w, i, 1}(x) & p_{w, i, 1}(x)>0 \\
0 & p_{w, i, 1}(x) \leq 0
\end{array}, \quad i \geq 0 .\right.
\end{aligned}
$$

If we define $V_{i}=\left\{x \in[0, \infty) \mid p_{w, i, 1}(x)>0\right\}$ and $W_{i}=\left\{x \in[0, \infty) \mid p_{w, i, 1}(x) \leq 0\right\}$ for $i=0,1,2, \ldots$, then for $\left(p_{I}, p_{w}\right) \in D(A)$, we calculate

$$
\begin{aligned}
& \int_{0}^{\infty} \frac{d p_{w, i, 1}(x)}{d x} \frac{\left[p_{w, i, 1}(x)\right]^{+}}{p_{w, i, 1}(x)} d x \\
& \quad=\int_{V_{i}} \frac{d p_{w, i, 1}(x)}{d x} \frac{\left[p_{w, i, 1}(x)\right]^{+}}{p_{w, i, 1}(x)} d x+\int_{W_{i}} \frac{d p_{w, i, 1}(x)}{d x} \frac{\left[p_{w, i, 1}(x)\right]^{+}}{p_{w, i, 1}(x)} d x
\end{aligned}
$$




$$
\begin{aligned}
& =\int_{V_{i}} \frac{d p_{w, i, 1}(x)}{d x} d x=\int_{0}^{\infty} \frac{d\left[p_{w, i, 1}(x)\right]^{+}}{d x} d x \\
& =\left.\left[p_{w, i, 1}(x)\right]^{+}\right|_{0} ^{\infty}=-\left[p_{w, i, 1}(0)\right]^{+}, \quad i \geq 0 .
\end{aligned}
$$

By using the boundary conditions on $\left(p_{I}, p_{w}\right) \in D(A)$ and (2.12) for such $(\phi, \psi)$, we derive

$$
\begin{aligned}
& \left\langle(A+U+E)\left(p_{I}, p_{w}\right),(\phi, \psi)\right\rangle \\
& =\left(-\lambda p_{I, 0,0}+\int_{0}^{\infty} \mu(x) p_{w, 0,1}(x) d x\right) \frac{\left[p_{I, 0,0}\right]^{+}}{p_{I, 0,0}} \\
& +\sum_{i=1}^{\infty}\left[-(\lambda+i \theta) p_{I, i, 0}+\int_{0}^{\infty} \mu(x) p_{w, i, 1}(x) d x\right] \frac{\left[p_{I, i, 0}\right]^{+}}{p_{I, i, 0}} \\
& +\int_{0}^{\infty}\left\{-\frac{d p_{w, 0,1}(x)}{d x}-(\lambda+\alpha+\mu(x)) p_{w, 0,1}(x)\right\} \frac{\left[p_{w, 0,1}(x)\right]^{+}}{p_{w, 0,1}(x)} d x \\
& +\sum_{i=1}^{\infty} \int_{0}^{\infty}\left\{-\frac{d p_{w, i, 1}(x)}{d x}-(\lambda+\alpha+\mu(x)) p_{w, i, 1}(x)\right. \\
& \left.+\lambda p_{w, i-1,1}(x)\right\} \frac{\left[p_{w, i, 1}(x)\right]^{+}}{p_{w, i, 1}(x)} d x \\
& =-\lambda\left[p_{I, 0,0}\right]^{+}+\frac{\left[p_{I, 0,0}\right]^{+}}{p_{I, 0,0}} \int_{0}^{\infty} \mu(x) p_{w, 0,1}(x) d x \\
& -\sum_{i=1}^{\infty}(\lambda+i \theta)\left[p_{I, i, 0}\right]^{+}+\sum_{i=1}^{\infty} \frac{\left[p_{I, i, 0}\right]^{+}}{p_{I, i, 0}} \int_{0}^{\infty} \mu(x) p_{w, i, 1}(x) d x \\
& +\int_{0}^{\infty}-\frac{d p_{w, 0,1}(x)}{d x} \frac{\left[p_{w, 0,1}(x)\right]^{+}}{p_{w, 0,1}(x)} d x \\
& -\int_{0}^{\infty}(\lambda+\alpha+\mu(x))\left[p_{w, 0,1}(x)\right]^{+} d x
\end{aligned}
$$




$$
\begin{aligned}
& +\sum_{i=1}^{\infty} \int_{0}^{\infty}-\frac{d p_{w, i, 1}(x)}{d x} \frac{\left[p_{w, i, 1}(x)\right]^{+}}{p_{w, i, 1}(x)} d x \\
& -\sum_{i=1}^{\infty} \int_{0}^{\infty}(\lambda+\alpha+\mu(x))\left[p_{w, i, 1}(x)\right]^{+} d x \\
& +\sum_{i=1}^{\infty} \int_{0}^{\infty} \lambda p_{w, i-1,1}(x) \frac{\left[p_{w, i, 1}(x)\right]^{+}}{p_{w, i, 1}(x)} d x \\
& =-\sum_{i=0}^{\infty}(\lambda+i \theta)\left[p_{I, i, 0}\right]^{+}+\sum_{i=0}^{\infty} \frac{\left[p_{I, i, 0}\right]^{+}}{p_{I, i, 0}} \int_{0}^{\infty} \mu(x) p_{w, i, 1}(x) d x \\
& -\sum_{i=0}^{\infty} \int_{0}^{\infty} \frac{d p_{w, i, 1}(x)}{d x} \frac{\left[p_{w, i, 1}(x)\right]^{+}}{p_{w, i, 1}(x)} d x \\
& -\sum_{i=0}^{\infty} \int_{0}^{\infty}(\lambda+\alpha+\mu(x))\left[p_{w, i, 1}(x)\right]^{+} d x \\
& +\sum_{i=1}^{\infty} \int_{0}^{\infty} \lambda p_{w, i-1,1}(x) \frac{\left[p_{w, i, 1}(x)\right]^{+}}{p_{w, i, 1}(x)} d x \\
& =-\sum_{i=0}^{\infty}(\lambda+i \theta)\left[p_{I, i, 0}\right]^{+}+\sum_{i=0}^{\infty} \frac{\left[p_{I, i, 0}\right]^{+}}{p_{I, i, 0}} \int_{0}^{\infty} \mu(x) p_{w, i, 1}(x) d x \\
& +\sum_{i=0}^{\infty}\left[p_{w, i, 1}(0)\right]^{+}-\sum_{i=0}^{\infty} \int_{0}^{\infty}(\lambda+\alpha+\mu(x))\left[p_{w, i, 1}(x)\right]^{+} d x \\
& +\sum_{i=1}^{\infty} \int_{0}^{\infty} \lambda p_{w, i-1,1}(x) \frac{\left[p_{w, i, 1}(x)\right]^{+}}{p_{w, i, 1}(x)} d x \\
& =-\sum_{i=0}^{\infty}(\lambda+i \theta)\left[p_{I, i, 0}\right]^{+}+\sum_{i=0}^{\infty} \frac{\left[p_{I, i, 0}\right]^{+}}{p_{I, i, 0}} \int_{0}^{\infty} \mu(x) p_{w, i, 1}(x) d x \\
& +\sum_{i=0}^{\infty}\left[\lambda p_{I, i, 0}+(i+1) \theta p_{I, i+1,0}\right]^{+}
\end{aligned}
$$




$$
\begin{aligned}
& -\sum_{i=0}^{\infty} \int_{0}^{\infty}(\lambda+\alpha+\mu(x))\left[p_{w, i, 1}(x)\right]^{+} d x \\
& +\sum_{i=1}^{\infty} \int_{0}^{\infty} \lambda p_{w, i-1,1}(x) \frac{\left[p_{w, i, 1}(x)\right]^{+}}{p_{w, i, 1}(x)} d x \\
& \leq-\sum_{i=0}^{\infty}(\lambda+i \theta)\left[p_{I, i, 0}\right]^{+}+\sum_{i=0}^{\infty} \frac{\left[p_{I, i, 0}\right]^{+}}{p_{I, i, 0}} \int_{0}^{\infty} \mu(x) p_{w, i, 1}(x) d x \\
& +\sum_{i=0}^{\infty}\left\{\lambda\left[p_{I, i, 0}\right]^{+}+(i+1) \theta\left[p_{I, i+1,0}\right]^{+}\right\} \\
& -\sum_{i=0}^{\infty} \int_{0}^{\infty}(\lambda+\alpha+\mu(x))\left[p_{w, i, 1}(x)\right]^{+} d x \\
& +\sum_{i=1}^{\infty} \int_{0}^{\infty} \lambda\left[p_{w, i-1,1}(x)\right]^{+} d x \\
& =-\sum_{i=0}^{\infty}(\lambda+i \theta)\left[p_{I, i, 0}\right]^{+}+\sum_{i=0}^{\infty} \lambda\left[p_{I, i, 0}\right]^{+}+\sum_{i=0}^{\infty}(i+1) \theta\left[p_{I, i+1,0}\right]^{+} \\
& +\sum_{i=0}^{\infty} \frac{\left[p_{I, i, 0}\right]^{+}}{p_{I, i, 0}} \int_{0}^{\infty} \mu(x)\left[p_{w, i, 1}(x)\right]^{+} d x \\
& -\sum_{i=0}^{\infty} \int_{0}^{\infty}(\lambda+\alpha+\mu(x))\left[p_{w, i, 1}(x)\right]^{+} d x \\
& +\sum_{i=0}^{\infty} \int_{0}^{\infty} \lambda\left[p_{w, i, 1}(x)\right]^{+} d x \\
& =-\sum_{i=0}^{\infty} i \theta\left[p_{I, i, 0}\right]^{+}+\sum_{i=0}^{\infty}(i+1) \theta\left[p_{I, i+1,0}\right]^{+} \\
& +\sum_{i=0}^{\infty}\left(\frac{\left[p_{I, i, 0}\right]^{+}}{p_{I, i, 0}}-1\right) \int_{0}^{\infty} \mu(x)\left[p_{w, i, 1}(x)\right]^{+} d x
\end{aligned}
$$




$$
\begin{aligned}
& -\alpha \sum_{i=0}^{\infty} \int_{0}^{\infty}\left[p_{w, i, 1}(x)\right]^{+} d x \\
= & -\sum_{i=0}^{\infty}(i+1) \theta\left[p_{I, i+1,0}\right]^{+}+\sum_{i=0}^{\infty}(i+1) \theta\left[p_{I, i+1,0}\right]^{+} \\
& +\sum_{i=0}^{\infty}\left(\frac{\left[p_{I, i, 0}\right]^{+}}{p_{I, i, 0}}-1\right) \int_{0}^{\infty} \mu(x)\left[p_{w, i, 1}(x)\right]^{+} d x \\
& -\alpha \sum_{i=0}^{\infty} \int_{0}^{\infty}\left[p_{w, i, 1}(x)\right]^{+} d x \\
= & \sum_{i=0}^{\infty}\left(\frac{\left[p_{I, i, 0}\right]^{+}}{p_{I, i, 0}}-1\right) \int_{0}^{\infty} \mu(x)\left[p_{w, i, 1}(x)\right]^{+} d x \\
& -\alpha \sum_{i=0}^{\infty} \int_{0}^{\infty}\left[p_{w, i, 1}(x)\right]^{+} d x \\
\leq & 0 .
\end{aligned}
$$

In the above, we have used the following inequalities:

$$
\begin{aligned}
& \int_{0}^{\infty} p_{w, i-1,1}(x) \frac{\left[p_{w, i, 1}(x)\right]^{+}}{p_{w, i, 1}(x)} d x \\
& \quad \leq \int_{0}^{\infty}\left[p_{w, i-1,1}(x)\right]^{+} \frac{\left[p_{w, i, 1}(x)\right]^{+}}{p_{w, i, 1}(x)} d x \\
& \quad \leq \int_{0}^{\infty}\left[p_{w, i-1,1}(x)\right]^{+} d x, \quad i \geq 1 .
\end{aligned}
$$

(2.13) shows that $A+U+E$ is dispersive.

From the first step, second step and the Hille-Yosida theorem (see Gupur et al. [7]), we know that $A$ generates a $C_{0}$-semigroup. By using the perturbation theorem of a $C_{0}$-semigroup and the third step, we deduce that $A+U+E$ generates a $C_{0}$-semigroup $T(t)$. By combining the first step, second step, fourth step and the Phillips theorem, we derive that $A+U+E$ generates a positive contraction $C_{0}$-semigroup (see Gupur et al. [7]). By the uniqueness theorem of a $C_{0}$-semigroup we know that this semigroup is just $T(t)$. 
Theorem 2.2 If $\mu=\sup _{x \in[0, \infty)} \mu(x)<\infty$, then the system (1.6) has a unique nonnegative time-dependent solution $\left(p_{I}, p_{w}\right)(x, t)$ satisfying $\left\|\left(p_{I}, p_{w}\right)(\cdot, t)\right\| \leq 1, \forall t \in$ $[0, \infty)$.

Proof Since the initial value $\left(p_{I}, p_{w}\right)(0)$ of the system (1.6) belongs to $D(A)$, from Theorem 2.1 and Gupur et al. [7], we know that the system (1.6) has a unique nonnegative time-dependent solution $\left(p_{I}, p_{w}\right)(x, t)$, which can be expressed by

$$
\left(p_{I}, p_{w}\right)(x, t)=T(t)\left(p_{I}, p_{w}\right)(0), \quad \forall t \in[0, \infty) .
$$

Because $T(t)$ is contractive by Theorem 2.1, i.e., $\|T(t)\| \leq 1$, by (2.14) it follows that

$$
\begin{aligned}
\left\|\left(p_{I}, p_{w}\right)(\cdot, t)\right\| & =\left\|T(t)\left(p_{I}, p_{w}\right)(0)\right\| \leq\|T(t)\|\left\|\left(p_{I}, p_{w}\right)(0)\right\| \\
& \leq\left\|\left(p_{I}, p_{w}\right)(0)\right\|=1, \quad \forall t \in[0, \infty) .
\end{aligned}
$$

This reflects the physical meaning of $\left(p_{I}, p_{w}\right)(x, t)$.

Remark 2.3 It is not difficult to verify that $T(t)$ is not isometric, so we cannot obtain $\left\|\left(p_{I}, p_{w}\right)(\cdot, t)\right\|=1, \quad \forall t \in[0, \infty)$. This is different from Gupur [6].

\section{Asymptotic behavior of the time-dependent solution of the system (1.6) When $\mu(x)=\mu$}

In this section, firstly we determine the expression of $(A+U+E)^{*}$, the adjoint operator of $A+U+E$, next we study the resolvent set of $(A+U+E)^{*}$, through which we deduce the resolvent set of $A+U+E$ on the imaginary axis. Thirdly, we prove that 0 is not an eigenvalue of $A+U+E$, which means that the system (1.6) has only the zero steady-state solution and therefore the hypothesis 2 does not hold.

It is easy to see that $X^{*}$, the dual space of $X$, is

$$
X^{*}=\left\{\begin{array}{l|l}
\left(q_{I}^{*}, q_{w}^{*}\right) \mid \begin{array}{c}
q_{I}^{*}=\left(q_{I, 0,0}^{*}, q_{I, 1,0}^{*}, q_{I, 2,0}^{*}, \ldots\right) \in l^{\infty} \\
q_{w}^{*}=\left(q_{w, 0,1}^{*}, q_{w, 1,1}^{*}, q_{w, 2,1}^{*}, \ldots\right) \\
\in L^{\infty}[0, \infty) \times L^{\infty}[0, \infty) \times L^{\infty}[0, \infty) \times \cdots, \\
\left\|\left(q_{I}^{*}, q_{w}^{*}\right)\right\|=\sup \left\{\sup _{i \geq 0}\left|q_{I, i, 0}^{*}\right|, \sup _{i \geq 0}\left\|q_{w, i, 1}^{*}\right\|_{L^{\infty}[0, \infty)}\right. \\
<\infty
\end{array}
\end{array}\right\} .
$$

It is obvious that $X^{*}$ is a Banach space.

Lemma 3.1 $(A+U+E)^{*}$, the adjoint operator of $A+U+E$, is as follows.

$$
(A+U+E)^{*}\left(q_{I}^{*}, q_{w}^{*}\right)=(L+G+H)\left(q_{I}^{*}, q_{w}^{*}\right), \quad \forall\left(q_{I}^{*}, q_{w}^{*}\right) \in D(L),
$$


where

$$
\begin{aligned}
& L\left(q_{I}^{*}, q_{w}^{*}\right)(x)=\left(\left(\begin{array}{cccc}
-\lambda & 0 & 0 & \cdots \\
0 & -(\lambda+\theta) & 0 & \cdots \\
0 & 0 & -(\lambda+2 \theta) & \cdots \\
\vdots & \vdots & \vdots & \vdots
\end{array}\right)\left(\begin{array}{c}
q_{I, 0,0}^{*} \\
q_{I, 1,0}^{*} \\
q_{I, 2,0}^{*} \\
\vdots
\end{array}\right)\right. \\
& \left.\left(\begin{array}{ccc}
\frac{d}{d x}-(\lambda+\alpha+\mu) & 0 & \cdots \\
0 & \frac{d}{d x}-(\lambda+\alpha+\mu) & \cdots \\
\vdots & \vdots & \vdots
\end{array}\right)\left(\begin{array}{c}
q_{w, 0,1}^{*}(x) \\
q_{w, 1,1}^{*}(x) \\
\vdots
\end{array}\right)\right) \text {, } \\
& G\left(q_{I}^{*}, q_{w}^{*}\right)(x)=\left(\left(\begin{array}{c}
0 \\
0 \\
0 \\
\vdots
\end{array}\right),\left(\begin{array}{cccc}
\mu & 0 & 0 & \cdots \\
0 & \mu & 0 & \cdots \\
0 & 0 & \mu & \cdots \\
\vdots & \vdots & \vdots & \vdots
\end{array}\right)\left(\begin{array}{c}
q_{I, 0,0}^{*} \\
q_{I, 1,0}^{*} \\
q_{I, 2,0}^{*} \\
\vdots
\end{array}\right)\right) \text {, } \\
& H\left(q_{I}^{*}, q_{w}^{*}\right)(x)=\left(\left(\begin{array}{ccccc}
\lambda & 0 & 0 & 0 & \cdots \\
\theta & \lambda & 0 & 0 & \cdots \\
0 & 2 \theta & \lambda & 0 & \ldots \\
0 & 0 & 3 \theta & \lambda & \ldots \\
\vdots & \vdots & \vdots & \vdots & \vdots
\end{array}\right)\left(\begin{array}{c}
q_{w, 0,1}^{*}(0) \\
q_{w, 1,1}^{*}(0) \\
q_{w, 2,1}^{*}(0) \\
q_{w, 3,1}^{*}(0) \\
\vdots
\end{array}\right)\right. \text {, } \\
& \left.\left(\begin{array}{cccccc}
0 & \lambda & 0 & 0 & 0 & \cdots \\
0 & 0 & \lambda & 0 & 0 & \cdots \\
0 & 0 & 0 & \lambda & 0 & \cdots \\
0 & 0 & 0 & 0 & \lambda & \cdots \\
\vdots & \vdots & \vdots & \vdots & \vdots & \vdots
\end{array}\right)\left(\begin{array}{c}
q_{w, 0,1}^{*}(x) \\
q_{w, 1,1}^{*}(x) \\
q_{w, 2,1}^{*}(x) \\
q_{w, 3,1}^{*}(x) \\
\vdots
\end{array}\right)\right), \\
& D(L)=\left\{\begin{array}{l|l}
\left(q_{I}^{*}, q_{w}^{*}\right) \in X^{*} & \begin{array}{c}
q_{w, i, 1}^{*}(x) \text { are absolutely continuous } \\
\text { and satisfy } q_{w, i, 1}^{*}(\infty)=\eta, i \geq 0
\end{array}
\end{array}\right\}, \\
& D(G)=D(H)=X^{*} .
\end{aligned}
$$

Here $\eta$ in $D(L)$ is a constant which is irrelevant to $i$.

Proof For any $\left(p_{I}, p_{w}\right) \in D(A)$ and $\left(q_{i}^{*}, q_{w}^{*}\right) \in D(L)$, by using the boundary conditions on $\left(p_{I}, p_{w}\right) \in D(A)$ and integration by parts, we have

$$
\begin{aligned}
& \left\langle(A+U+E)\left(p_{I}, p_{w}\right),\left(q_{I}^{*}, q_{w}^{*}\right)\right\rangle \\
& =\sum_{i=0}^{\infty}\left\{-(\lambda+i \theta) p_{I, i, 0}+\mu \int_{0}^{\infty} p_{w, i, 1}(x) d x\right\} q_{I, i, 0}^{*} \\
& \quad+\int_{0}^{\infty}\left\{-\frac{d p_{w, 0,1}(x)}{d x}-(\lambda+\alpha+\mu) p_{w, 0,1}(x)\right\} q_{w, 0,1}^{*}(x) d x
\end{aligned}
$$




$$
\begin{aligned}
& +\sum_{i=1}^{\infty} \int_{0}^{\infty}\left\{-\frac{d p_{w, i, 1}(x)}{d x}-(\lambda+\alpha+\mu) p_{w, i, 1}(x)\right. \\
& \left.+\lambda p_{w, i-1,1}(x)\right\} q_{w, i, 1}^{*}(x) d x \\
& =\sum_{i=0}^{\infty}-(\lambda+i \theta) p_{I, i, 0} q_{I, i, 0}^{*}+\sum_{i=0}^{\infty} \int_{0}^{\infty} \mu p_{w, i, 1}(x) q_{I, i, 0}^{*} d x \\
& +\sum_{i=0}^{\infty} \int_{0}^{\infty}-\frac{d p_{w, i, 1}(x)}{d x} q_{w, i, 1}^{*}(x) d x \\
& +\sum_{i=0}^{\infty} \int_{0}^{\infty}-(\lambda+\alpha+\mu) p_{w, i, 1}(x) q_{w, i, 1}^{*}(x) d x \\
& +\sum_{i=1}^{\infty} \int_{0}^{\infty} \lambda p_{w, i-1,1}(x) q_{w, i, 1}^{*}(x) d x \\
& =\sum_{i=0}^{\infty}-(\lambda+i \theta) p_{I, i, 0} q_{I, i, 0}^{*}+\sum_{i=0}^{\infty} \int_{0}^{\infty} \mu p_{w, i, 1}(x) q_{I, i, 0}^{*} d x \\
& -\left.\sum_{i=0}^{\infty} p_{w, i, 1}(x) q_{w, i, 1}^{*}(x)\right|_{0} ^{\infty}+\sum_{i=0}^{\infty} \int_{0}^{\infty} p_{w, i, 1}(x) \frac{d q_{w, i, 1}^{*}(x)}{d x} d x \\
& +\sum_{i=0}^{\infty} \int_{0}^{\infty}-(\lambda+\alpha+\mu) p_{w, i, 1}(x) q_{w, i, 1}^{*}(x) d x \\
& +\sum_{i=0}^{\infty} \int_{0}^{\infty} \lambda p_{w, i, 1}(x) q_{w, i+1,1}^{*}(x) d x \\
& =\sum_{i=0}^{\infty}-(\lambda+i \theta) p_{I, i, 0} q_{I, i, 0}^{*}+\sum_{i=0}^{\infty} \int_{0}^{\infty} \mu p_{w, i, 1}(x) q_{I, i, 0}^{*} d x \\
& +\sum_{i=0}^{\infty} p_{w, i, 1}(0) q_{w, i, 1}^{*}(0) \\
& +\sum_{i=0}^{\infty} \int_{0}^{\infty} p_{w, i, 1}(x)\left\{\frac{d q_{w, i, 1}^{*}(x)}{d x}-(\lambda+\alpha+\mu) q_{w, i, 1}^{*}(x)\right\} d x
\end{aligned}
$$




$$
\begin{aligned}
& +\sum_{i=0}^{\infty} \int_{0}^{\infty} \lambda p_{w, i, 1}(x) q_{w, i+1,1}^{*}(x) d x \\
= & \sum_{i=0}^{\infty}-(\lambda+i \theta) p_{I, i, 0} q_{I, i, 0}^{*}+\sum_{i=0}^{\infty} \int_{0}^{\infty} \mu p_{w, i, 1}(x) q_{I, i, 0}^{*} d x \\
& +\sum_{i=0}^{\infty}\left[\lambda p_{I, i, 0}+(i+1) \theta p_{I, i+1,0}\right] q_{w, i, 1}^{*}(0) \\
& +\sum_{i=0}^{\infty} \int_{0}^{\infty} p_{w, i, 1}(x)\left\{\frac{d q_{w, i, 1}^{*}(x)}{d x}-(\lambda+\alpha+\mu) q_{w, i, 1}^{*}(x)\right\} d x \\
& +\sum_{i=0}^{\infty} \int_{0}^{\infty} \lambda p_{w, i, 1}(x) q_{w, i+1,1}^{*}(x) d x \\
= & \left\langle\left(p_{I}, p_{w}^{\infty}\right),(L+G+H)\left(q_{I}^{*}, q_{w}^{*}\right)\right\rangle . \\
& +\sum_{i=0}^{\infty}-(\lambda+i \theta) p_{I, i, 0} q_{I, i, 0}^{*} p_{I, i, 0} \lambda q_{w, i, 1}^{*}(0)+\sum_{i=0}^{\infty} p_{I, i+1,0}(i+1) \theta q_{w, i, 1}^{*}(0) \\
& +\sum_{i=0}^{\infty} \int_{0}^{\infty} \int_{0}^{\infty} p_{w, i, 1}(x) \mu q_{I, i, 0}^{*} d x \\
& \left.+\frac{d q_{w, i, 1}^{*}(x)}{d x}-(\lambda+\alpha+\mu) q_{w, i, 1}^{*}(x)\right\} d x
\end{aligned}
$$

(3.1) shows that the result of this lemma is right.

\section{Lemma 3.2}

$$
\left\{\begin{array}{l|l}
\gamma \in \mathbb{C} & \begin{array}{l}
\sup \left\{\frac{\lambda}{|\gamma+\lambda|}, \sup _{i \geq 1}\left\{\frac{\lambda+i \theta}{|\gamma+\lambda+i \theta|}\right\}, \frac{\lambda \mu}{|\gamma+\lambda||\gamma+\lambda+\alpha+\mu|}+\frac{\lambda}{\operatorname{Re} \gamma+\lambda+\alpha+\mu},\right. \\
\left.\sup _{i \geq 1}\left\{\frac{\mu(\lambda+i \theta)}{|(\gamma+\lambda+\alpha+\mu)(\gamma+\lambda+i \theta)|}+\frac{\lambda}{\operatorname{Re} \gamma+\lambda+\alpha+\mu}\right\}\right\}<1, \\
\operatorname{Re} \gamma+\lambda+\alpha+\mu>0
\end{array}
\end{array}\right\}
$$


belongs to the resolvent set of $(A+U+E)^{*}$. In particular, all points on the imaginary axis except zero belong to the resolvent set of $(A+U+E)^{*}$, which implies that all points on the imaginary axis except zero belong to the resolvent set of $A+U+E$.

Proof For any given $\left(y_{I}^{*}, y_{w}^{*}\right) \in X^{*}$, consider the equation $(\gamma I-L-G)\left(q_{I}^{*}, q_{w}^{*}\right)=$ $H\left(y_{I}^{*}, y_{w}^{*}\right)$, that is,

$$
\begin{aligned}
& (\gamma+\lambda) q_{I, 0,0}^{*}=\lambda y_{w, 0,1}^{*}(0), \\
& (\gamma+\lambda+\theta) q_{I, 1,0}^{*}=\theta y_{w, 0,1}^{*}(0)+\lambda y_{w, 1,1}^{*}(0), \\
& \ldots \ldots \\
& (\gamma+\lambda+i \theta) q_{I, i, 0}^{*}=i \theta y_{w, i-1,1}^{*}(0)+\lambda y_{w, i, 1}^{*}(0), \quad i=1,2,3, \ldots, \\
& \frac{d q_{w, i, 1}^{*}(x)}{d x}=(\gamma+\lambda+\alpha+\mu) q_{w, i, 1}^{*}(x)-\mu q_{I, i, 0}^{*}-\lambda y_{w, i+1,1}^{*}(x), \quad i \geq 0, \\
& \ldots \ldots \\
& q_{w, i, 1}^{*}(\infty)=\eta, \quad i=0,1,2, \ldots
\end{aligned}
$$

By solving (3.2)-(3.5), we have

$$
\begin{gathered}
q_{I, 0,0}^{*}=\frac{\lambda}{\gamma+\lambda} y_{w, 0,1}^{*}(0), \\
q_{I, i, 0}^{*}=\frac{i \theta}{\gamma+\lambda+i \theta} y_{w, i-1,1}^{*}(0)+\frac{\lambda}{\gamma+\lambda+i \theta} y_{w, i, 1}^{*}(0), \quad i \geq 1, \\
q_{w, i, 1}^{*}(x)=b_{i} e^{(\gamma+\lambda+\alpha+\mu) x} \\
+e^{(\gamma+\lambda+\alpha+\mu) x} \int_{0}^{x}\left[-\mu q_{I, i, 0}^{*}-\lambda y_{w, i+1,1}^{*}(\tau)\right] e^{-(\gamma+\lambda+\alpha+\mu) \tau} d \tau \\
=b_{i} e^{(\gamma+\lambda+\alpha+\mu) x} \\
-e^{(\gamma+\lambda+\alpha+\mu) x} \int_{0}^{x}\left[\mu q_{I, i, 0}^{*}+\lambda y_{w, i+1,1}^{*}(\tau)\right] e^{-(\gamma+\lambda+\alpha+\mu) \tau} d \tau, \\
i \geq 0 .
\end{gathered}
$$

Through multiplying $e^{-(\gamma+\lambda+\alpha+\mu) x}$ to two side of (3.9), we calculate

$$
\begin{gathered}
e^{-(\gamma+\lambda+\alpha+\mu) x} q_{w, i, 1}^{*}(x)=b_{i}-\int_{0}^{x}\left[\mu q_{I, i, 0}^{*}+\lambda y_{w, i+1,1}^{*}(\tau)\right] e^{-(\gamma+\lambda+\alpha+\mu) \tau} d \tau, \\
i \geq 0 .
\end{gathered}
$$


By combining (3.6) with (3.10), we deduce, without loss of generality assuming $\operatorname{Re}(\gamma+\lambda+\alpha+\mu)>0$,

$$
b_{i}=\int_{0}^{\infty}\left[\mu q_{I, i, 0}^{*}+\lambda y_{w, i+1,1}^{*}(x)\right] e^{-(\gamma+\lambda+\alpha+\mu) x} d x, \quad i \geq 0 .
$$

By inserting (3.11) into (3.9), it follows that

$$
\begin{aligned}
q_{w, i, 1}^{*}(x)= & e^{(\gamma+\lambda+\alpha+\mu) x} \int_{x}^{\infty}\left[\mu q_{I, i, 0}^{*}+\lambda y_{w, i+1,1}^{*}(\tau)\right] e^{-(\gamma+\lambda+\alpha+\mu) \tau} d \tau \\
= & \frac{\mu}{\gamma+\lambda+\alpha+\mu} q_{I, i, 0}^{*} \\
& +\lambda e^{(\gamma+\lambda+\alpha+\mu) x} \int_{x}^{\infty} y_{w, i+1,1}^{*}(\tau) e^{-(\gamma+\lambda+\alpha+\mu) \tau} d \tau \\
\Longrightarrow & \frac{\mu}{|\gamma+\lambda+\alpha+\mu|}\left|q_{I, i, 0}^{*}\right| \\
\left\|q_{w, i, 1}^{*}\right\|_{L^{\infty}[0, \infty)} & +\frac{\lambda}{\operatorname{Re} \gamma+\lambda+\alpha+\mu}\left\|y_{w, i+1,1}^{*}\right\|_{L^{\infty}[0, \infty), \quad i \geq 0}
\end{aligned}
$$

(3.7) gives

$$
\left|q_{I, 0,0}^{*}\right| \leq \frac{\lambda}{|\gamma+\lambda|}\left|y_{w, 0,1}^{*}(0)\right| \leq \frac{\lambda}{|\gamma+\lambda|}\left\|y_{w, 0,1}\right\|_{L^{\infty}[0, \infty)} .
$$

By (3.8) we estimate

$$
\begin{aligned}
\left|q_{I, i, 0}^{*}\right| & \leq \frac{i \theta}{|\gamma+\lambda+i \theta|}\left|y_{w, i-1,1}^{*}(0)\right|+\frac{\lambda}{|\gamma+\lambda+i \theta|}\left|y_{w, i, 1}^{*}(0)\right| \\
& \leq \frac{i \theta}{|\gamma+\lambda+i \theta|}\left\|y_{w, i-1,1}^{*}\right\|_{L^{\infty}[0, \infty)}+\frac{\lambda}{|\gamma+\lambda+i \theta|}\left\|y_{w, i, 1}^{*}\right\|_{L^{\infty}[0, \infty)} \\
& \leq \frac{\lambda+i \theta}{|\gamma+\lambda+i \theta|} \sup _{i \geq 0}\left\|y_{w, i, 1}\right\|_{L^{\infty}[0, \infty)}, \quad i \geq 1
\end{aligned}
$$

By inserting (3.14) and (3.15) into (3.13), we have

$$
\begin{aligned}
\left\|q_{w, i, 1}^{*}\right\|_{L^{\infty}[0, \infty)} \leq & \frac{\mu}{|\gamma+\lambda+\alpha+\mu|} \frac{\lambda+i \theta}{|\gamma+\lambda+i \theta|} \sup _{i \geq 0}\left\|y_{w, i, 1}^{*}\right\|_{L^{\infty}[0, \infty)} \\
& +\frac{\lambda}{\operatorname{Re} \gamma+\lambda+\alpha+\mu} \sup _{i \geq 0}\left\|y_{w, i, 1}^{*}\right\|_{L^{\infty}[0, \infty)}
\end{aligned}
$$




$$
\begin{aligned}
= & \left\{\frac{\mu(\lambda+i \theta)}{|\gamma+\lambda+\alpha+\mu||\gamma+\lambda+i \theta|}+\frac{\lambda}{\operatorname{Re} \gamma+\lambda+\alpha+\mu}\right\} \\
& \times \sup _{i \geq 0}\left\|y_{w, i, 1}^{*}\right\|_{L^{\infty}[0, \infty), \quad i \geq 1} \\
\left\|q_{w, 0,1}^{*}\right\|_{L^{\infty}[0, \infty) \leq} \leq & \frac{\mu}{|\gamma+\lambda+\alpha+\mu|} \frac{\lambda}{|\gamma+\lambda|}\left\|y_{w, 0,1}^{*}\right\|_{L^{\infty}[0, \infty)} \\
& +\frac{\lambda}{\operatorname{Re} \gamma+\lambda+\alpha+\mu}\left\|y_{w, 1,1}^{*}\right\|_{L^{\infty}[0, \infty)} \\
\leq & \left\{\frac{\lambda \mu}{|\gamma+\lambda||\gamma+\lambda+\alpha+\mu|}+\frac{\lambda}{\operatorname{Re} \gamma+\lambda+\alpha+\mu}\right\} \\
& \quad \sup _{i \geq 0}\left\|y_{w, i, 1}^{*}\right\|_{L^{\infty}[0, \infty) .}
\end{aligned}
$$

By combining (3.14) and (3.15) with (3.16) and (3.17), we derive

$$
\begin{aligned}
& \left\|\left(q_{I}^{*}, q_{w}^{*}\right)\right\| \leq \sup \left\{\frac{\lambda}{|\gamma+\lambda|}, \sup _{i \geq 1}\left\{\frac{\lambda+i \theta}{|\gamma+\lambda+i \theta|}\right\},\right. \\
& \quad \frac{\lambda \mu}{|\gamma+\lambda||\gamma+\lambda+\alpha+\mu|}+\frac{\lambda}{\operatorname{Re} \gamma+\lambda+\alpha+\mu}, \\
& \left.\quad \sup _{i \geq 1}\left\{\frac{\mu(\lambda+i \theta)}{|(\gamma+\lambda+\alpha+\mu)(\gamma+\lambda+i \theta)|}+\frac{\lambda}{\operatorname{Re} \gamma+\lambda+\alpha+\mu}\right\}\right\} .
\end{aligned}
$$

This shows that $\left[I-(\gamma I-L-G)^{-1} H\right]^{-1}$ exists and is bounded when $\gamma$ belongs to the set

$$
\left\{\begin{array}{l|l}
\gamma \in \mathbb{C} & \left.\begin{array}{l}
\sup \left\{\frac{\lambda}{|\gamma+\lambda|}, \sup _{i \geq 1}\left\{\frac{\lambda+i \theta}{|\gamma+\lambda+i \theta|}\right\},\right. \\
\frac{\lambda \mu}{|\gamma+\lambda||\gamma+\lambda+\alpha+\mu|}+\frac{\lambda}{\operatorname{Re} \gamma+\lambda+\alpha+\mu}, \\
\sup _{i \geq 1}\left\{\frac{\mu(\lambda+i \theta)}{|(\gamma+\lambda+\alpha+\mu)(\gamma+\lambda+i \theta)|}+\frac{\lambda}{\operatorname{Re} \gamma+\lambda+\alpha+\mu}\right\} \\
\operatorname{Re} \gamma+\lambda+\alpha+\mu>0
\end{array}\right\}<1,
\end{array}\right\} .
$$

Through discussing the solution of the equation $(\gamma I-L-G)\left(q_{I}^{*}, q_{w}^{*}\right)=\left(y_{I}^{*}, y_{w}^{*}\right)$ for any given $\left(y_{I}^{*}, y_{w}^{*}\right) \in X^{*}$, it is not difficult to verify $(\gamma I-L-G)^{-1}$ exists and is bounded when $\gamma$ satisfies (3.19). Therefore, by the resolvent equation

$$
\begin{aligned}
{[\gamma I-(L+G+H)]^{-1} } & =\left\{(\gamma I-L-G)\left[I-(\gamma I-L-G)^{-1} H\right]\right\}^{-1} \\
& =\left[I-(\gamma I-L-G)^{-1} H\right]^{-1}(\gamma I-L-G)^{-1}
\end{aligned}
$$


we know that $(\gamma I-L-G-H)^{-1}$ exists and is bounded when $\gamma$ belongs to (3.19), which means that

$$
\left\{\begin{array}{l|l}
\gamma \in \mathbb{C} & \left.\begin{array}{l}
\sup \left\{\frac{\lambda}{|\gamma+\lambda|}, \sup _{i \geq 1}\left\{\frac{\lambda+i \theta}{|\gamma+\lambda+i \theta|}\right\},\right. \\
\frac{\lambda \mu}{|\gamma+\lambda||\gamma+\lambda+\alpha+\mu|}+\frac{\lambda}{\operatorname{Re} \gamma+\lambda+\alpha+\mu}, \\
\sup _{i \geq 1}\left\{\frac{\mu(\lambda+i \theta)}{|(\gamma+\lambda+\alpha+\mu)(\gamma+\lambda+i \theta)|}+\frac{\lambda}{\operatorname{Re} \gamma+\lambda+\alpha+\mu}\right\} \\
\operatorname{Re} \gamma+\lambda+\alpha+\mu>0
\end{array}\right\}<1,
\end{array}\right\}
$$

belongs to $\rho(L+G+H)$.

In particular, if $\gamma=i a, a \in \mathbb{R} \backslash\{0\}, i^{2}=-1$, then all $\gamma$ automatically belong to (3.19). In fact, by simple calculation, we have

$$
\begin{aligned}
& \frac{\lambda}{\sqrt{a^{2}+\lambda^{2}}}<1 \\
& \sup _{k \geq 1} \frac{\lambda+k \theta}{\sqrt{a^{2}+(\lambda+k \theta)^{2}}}<1 \text {. } \\
& \lambda \alpha>0 \Rightarrow \lambda \alpha+\lambda \mu>\lambda \mu \Rightarrow \lambda(\alpha+\mu)>\lambda \mu \\
& \Longrightarrow \\
& (\alpha+\mu) \sqrt{a^{2}+\lambda^{2}}>\lambda(\alpha+\mu)>\lambda \mu \\
& \lambda \mu(\lambda+\alpha+\mu)<(\alpha+\mu) \sqrt{a^{2}+\lambda^{2}}(\lambda+\alpha+\mu) \\
& <(\alpha+\mu) \sqrt{a^{2}+\lambda^{2}} \sqrt{a^{2}+(\lambda+\alpha+\mu)^{2}} \\
& \Longrightarrow \\
& \lambda \mu(\lambda+\alpha+\mu)+\lambda \sqrt{a^{2}+\lambda^{2}} \sqrt{a^{2}+(\lambda+\alpha+\mu)^{2}} \\
& <(\lambda+\alpha+\mu) \sqrt{a^{2}+\lambda^{2}} \sqrt{a^{2}+(\lambda+\alpha+\mu)^{2}} \\
& \Longrightarrow \\
& \frac{\lambda \mu}{\sqrt{a^{2}+\lambda^{2}} \sqrt{a^{2}+(\lambda+\alpha+\mu)^{2}}}+\frac{\lambda}{\lambda+\alpha+\mu}<1 . \\
& \alpha+\mu>\mu \\
& (\alpha+\mu)(\lambda+k \theta)(\lambda+\alpha+\mu)>\mu(\lambda+k \theta)(\lambda+\alpha+\mu) \\
& \Longrightarrow \\
& (\alpha+\mu) \sqrt{a^{2}+(\lambda+\alpha+\mu)^{2}} \sqrt{a^{2}+(\lambda+k \theta)^{2}}
\end{aligned}
$$




$$
\begin{aligned}
& >(\alpha+\mu)(\lambda+k \theta)(\lambda+\alpha+\mu)>\mu(\lambda+k \theta)(\lambda+\alpha+\mu) \\
& \Longrightarrow \\
& (\lambda+\alpha+\mu) \sqrt{a^{2}+(\lambda+\alpha+\mu)^{2}} \sqrt{a^{2}+(\lambda+k \theta)^{2}} \\
& >\mu(\lambda+k \theta)(\lambda+\alpha+\mu)+\lambda \sqrt{a^{2}+(\lambda+\alpha+\mu)^{2}} \sqrt{a^{2}+(\lambda+k \theta)^{2}} \\
& \Longrightarrow \\
& \sup _{k \geq 1}\left\{\frac{\mu(\lambda+k \theta)}{\sqrt{a^{2}+(\lambda+\alpha+\mu)^{2}} \sqrt{a^{2}+(\lambda+k \theta)^{2}}}+\frac{\lambda}{\lambda+\alpha+\mu}\right\}<1 . \\
& \lambda+\alpha+\mu>0 .
\end{aligned}
$$

The above five inequalities show that all points on the imaginary axis except zero belong to the resolvent set of $(A+U+E)^{*}$. From the relation between the spectrum of $A+U+E$ and the spectrum of $(A+U+E)^{*}$ we know that all points on the imaginary axis except zero belong to the resolvent set of $(A+U+E)$.

Lemma 3.3 If $\alpha+\mu>\lambda$, then 0 is not an eigenvalue of $A+U+E$.

Proof Consider the equation $(A+U+E)\left(p_{I}, p_{w}\right)=0$. It is equivalent to

$$
\begin{gathered}
\lambda p_{I, 0,0}=\mu \int_{0}^{\infty} p_{w, 0,1}(x) d x, \\
(\lambda+i \theta) p_{I, i, 0}=\mu \int_{0}^{\infty} p_{w, i, 1}(x) d x, \quad i \geq 1, \\
\frac{d p_{w, 0,1}(x)}{d x}=-(\lambda+\alpha+\mu) p_{w, 0,1}(x), \\
\frac{d p_{w, i, 1}(x)}{d x}=-(\lambda+\alpha+\mu) p_{w, i, 1}(x)+\lambda p_{w, i-1,1}(x), \quad i \geq 1, \\
p_{w, i, 1}(0)=\lambda p_{I, i, 0}+(i+1) \theta p_{I, i+1,0}, \quad i \geq 0 .
\end{gathered}
$$

Through solving (3.22)-(3.23), we have

$$
\begin{aligned}
p_{w, 0,1}(x)= & b_{0} e^{-(\lambda+\alpha+\mu) x}, \\
p_{w, i, 1}(x)= & b_{i} e^{-(\lambda+\alpha+\mu) x} \\
& +\lambda e^{-(\lambda+\alpha+\mu) x} \int_{0}^{x} p_{w, i-1,1}(\tau) e^{(\lambda+\alpha+\mu) \tau} d \tau, \quad i \geq 1 .
\end{aligned}
$$


By inserting (3.25) and (3.26) into (3.20) and (3.21), respectively, and using the Fubini theorem, it follows that

$$
\begin{aligned}
\lambda p_{I, 0,0}= & \frac{\mu}{\lambda+\alpha+\mu} b_{0} \Rightarrow b_{0}=\frac{\lambda(\lambda+\alpha+\mu)}{\mu} p_{I, 0,0}, \\
(\lambda+i \theta) p_{I, i, 0}= & \frac{\mu}{\lambda+\alpha+\mu} b_{i} \\
& +\lambda \mu \int_{0}^{\infty} e^{-(\lambda+\alpha+\mu) x} \int_{0}^{x} p_{w, i-1,1}(\tau) e^{(\lambda+\alpha+\mu) \tau} d \tau d x \\
= & \frac{\mu}{\lambda+\alpha+\mu} b_{i}+\frac{\lambda \mu}{\lambda+\alpha+\mu} \int_{0}^{\infty} p_{w, i-1,1}(\tau) d \tau \\
= & \frac{\mu}{\lambda+\alpha+\mu} b_{i}+\frac{\lambda}{\lambda+\alpha+\mu}[\lambda+(i-1) \theta] p_{I, i-1,0},
\end{aligned}
$$

Through combining (3.26) with (3.24), we obtain

$$
b_{i}=\lambda p_{I, i, 0}+(i+1) \theta p_{I, i+1,0}, \quad i \geq 0 .
$$

This together with (3.27) give

$$
b_{0}-\lambda p_{I, 0,0}=\theta p_{I, 1,0} \Rightarrow p_{I, 1,0}=\frac{\lambda(\lambda+\alpha)}{\mu \theta} p_{I, 0,0}
$$

By inserting (3.29) into (3.28), we deduce

$$
\begin{aligned}
(\lambda+i \theta) p_{I, i, 0}= & \frac{\mu}{\lambda+\alpha+\mu}\left[\lambda p_{I, i, 0}+(i+1) \theta p_{I, i+1,0}\right] \\
& +\frac{\lambda}{\lambda+\alpha+\mu}[\lambda+(i-1) \theta] p_{I, i-1,0} \\
\Longrightarrow & \lambda \mu p_{I, i, 0}+(i+1) \theta \mu p_{I, i+1,0} \\
& +\lambda[\lambda+(i-1) \theta] p_{I, i-1,0} \\
(\lambda+\alpha+\mu)(\lambda+i \theta) p_{I, i, 0}= & -\lambda[\lambda+(i-1) \theta] p_{I, i-1,0} \\
(i+1) \theta \mu p_{I, i+1,0}= & \frac{\lambda(\lambda+\alpha)+i \theta(\lambda+\alpha+\mu)}{(i+1) \theta \mu} p_{I, i, 0} \\
p_{I, i+1,0}= & -\frac{\lambda[\lambda+(i-1) \theta]}{(i+1) \theta \mu} p_{I, i-1,0}, \quad i \geq 1 .
\end{aligned}
$$


From (3.31) and (3.30), we derive

$$
\begin{aligned}
p_{I, 2,0} & =\frac{\lambda(\lambda+\alpha)+\theta(\lambda+\alpha+\mu)}{2 \theta \mu} p_{I, 1,0}-\frac{\lambda^{2}}{2 \theta \mu} p_{I, 0,0} \\
& =\frac{\lambda(\lambda+\alpha)+\theta(\lambda+\alpha+\mu)}{2 \theta \mu} \frac{\lambda(\lambda+\alpha)}{\mu \theta} p_{I, 0,0}-\frac{\lambda^{2}}{2 \theta \mu} p_{I, 0,0} \\
& =\frac{\lambda(\lambda+\alpha)[\lambda(\lambda+\alpha)+\theta(\lambda+\alpha+\mu)]-\lambda^{2} \mu \theta}{2(\mu \theta)^{2}} p_{I, 0,0} \\
& =\frac{\lambda\left[(\lambda+\alpha)^{2}(\lambda+\theta)+\mu \alpha \theta\right]}{2(\mu \theta)^{2}} p_{I, 0,0} .
\end{aligned}
$$

By using (3.31), (3.32) and (3.30), we calculate

$$
\begin{aligned}
p_{I, 3,0} & =\frac{\lambda(\lambda+\alpha)+2 \theta(\lambda+\alpha+\mu)}{3 \theta \mu} p_{I, 2,0}-\frac{\lambda(\lambda+\theta)}{3 \theta \mu} p_{I, 1,0} \\
& =\frac{(\lambda+\alpha)^{3}(\lambda+\theta)(\lambda+2 \theta)+\alpha \mu \theta(\lambda+\alpha)(3 \lambda+4 \theta)+2 \alpha(\mu \theta)^{2}}{3 !(\mu \theta)^{3}} \lambda p_{I, 0,0} .
\end{aligned}
$$

(3.25) and (3.27) give

$$
p_{w, 0,1}(x)=\frac{\lambda(\lambda+\alpha+\mu)}{\mu} p_{I, 0,0} e^{-(\lambda+\alpha+\mu) x} .
$$

It is difficult to determine the explicit expression of $p_{I, i, 0}$ and $p_{w, i, 1}(x)$ for all $i \geq$ 3 . In the following we apply another method to deduce our result in this lemma. Define the probability generating functions $Q_{1}(z)=\sum_{i=0}^{\infty} p_{I, i, 0} z^{i}$ and $Q_{2}(x, z)=$ $\sum_{i=0}^{\infty} p_{w, i, 1}(x) z^{i}$ for $|z|<1$. Then Theorem 2.2 implies that $Q_{1}(z)$ and $Q_{2}(x, z)$ are well-defined. From (3.20), (3.21) and the basic knowledge of power series, we have

$$
\begin{aligned}
& \lambda p_{I, 0,0}+\sum_{i=1}^{\infty}(\lambda+i \theta) p_{I, i, 0} z^{i}=\mu \int_{0}^{\infty} p_{w, 0,1}(x) d x+\sum_{i=1}^{\infty} \mu \int_{0}^{\infty} p_{w, i, 1}(x) d x z^{i} \\
& \Longrightarrow \\
& \lambda Q_{1}(z)+z \theta \frac{d Q_{1}(z)}{d z}=\mu \int_{0}^{\infty} Q_{2}(x, z) d x
\end{aligned}
$$


By (3.22)-(3.23), we deduce

$$
\begin{aligned}
\frac{\partial \sum_{i=0}^{\infty} p_{w, i, 1}(x) z^{i}}{\partial x} & =-\sum_{i=0}^{\infty}(\lambda+\alpha+\mu) p_{w, i, 1}(x) z^{i}+\lambda \sum_{i=1}^{\infty} p_{w, i-1,1}(x) z^{i} \\
\frac{\partial Q_{2}(x, z)}{\partial x} & =-(\lambda+\alpha+\mu) Q_{2}(x, z)+\lambda z Q_{2}(x, z) \\
& =(\lambda z-\lambda-\alpha-\mu) Q_{2}(x, z) \\
& \Longrightarrow \\
Q_{2}(x, z) & =Q_{2}(0, z) e^{(\lambda z-\lambda-\alpha-\mu) x} .
\end{aligned}
$$

(3.24) implies

$$
\begin{aligned}
Q_{2}(0, z) & =\sum_{i=0}^{\infty} p_{w, i, 1}(0) z^{i}=\sum_{i=0}^{\infty}\left[\lambda p_{I, i, 0}+(i+1) \theta p_{I, i+1,0}\right] z^{i} \\
& =\lambda \sum_{i=0}^{\infty} p_{I, i, 0} z^{i}+\theta \sum_{i=0}^{\infty}(i+1) p_{I, i+1,0} z^{i} \\
& =\lambda Q_{1}(z)+\theta \frac{d Q_{1}(z)}{d z} .
\end{aligned}
$$

By inserting (3.37) into (3.36) and then inserting (3.36) into (3.35), and using $\operatorname{Re}(\lambda z-$ $\lambda-\alpha-\mu)<0$, we calculate

$$
\begin{aligned}
& \lambda Q_{1}(z)+z \theta \frac{d Q_{1}(z)}{d z} \\
& =\mu \int_{0}^{\infty}\left[\lambda Q_{1}(z)+\theta \frac{d Q_{1}(z)}{d z}\right] e^{(\lambda z-\lambda-\alpha-\mu) x} d x \\
& =\frac{-\mu}{\lambda z-\lambda-\alpha-\mu}\left[\lambda Q_{1}(z)+\theta \frac{d Q_{1}(z)}{d z}\right] \\
& \Longrightarrow \\
& \left.\Longrightarrow \frac{1}{\Longrightarrow} \frac{\mu}{\lambda z-\lambda-\alpha-\mu}\right] \lambda Q_{1}(z)=\left[\frac{-\mu}{\lambda z-\lambda-\alpha-\mu}-z\right] \theta \frac{d Q_{1}(z)}{d z} \\
& \frac{d Q_{1}(z)}{Q_{1}(z)}=\frac{\lambda(\lambda z-\lambda-\alpha)}{\theta[-\mu-z(\lambda z-\lambda-\alpha-\mu)]} d z=\frac{\lambda(\lambda+\alpha-\lambda z)}{\theta[\mu+z(\lambda z-\lambda-\alpha-\mu)]} d z \\
& \quad \Longrightarrow \quad Q_{1}(z)=Q_{1}(0) e^{\int_{0}^{z} \frac{\lambda(\lambda+\alpha-\lambda \xi)}{\theta[\mu+\xi(\lambda \xi-\lambda-\alpha-\mu)]} d \xi} \\
& =p_{I, 0,0} e^{\int_{0}^{z} \frac{\lambda(\lambda+\alpha-\lambda \xi)}{\theta[\mu+\xi(\lambda \xi-\lambda-\alpha-\mu)]} d \xi} .
\end{aligned}
$$


This together with (3.36) and (3.37) give

$$
\begin{aligned}
Q_{2}(x, z)= & \lambda p_{I, 0,0}\left\{1+\frac{\lambda+\alpha-\lambda z}{\mu+z(\lambda z-\lambda-\alpha-\mu)}\right\} \\
& \times e^{\int_{0}^{z} \frac{\lambda(\lambda+\alpha-\lambda \xi)}{\theta[\mu+\xi(\lambda \xi-\lambda-\alpha-\mu)]} d \xi} e^{(\lambda z-\lambda-\alpha-\mu) x} .
\end{aligned}
$$

It is easy to see that $\xi=\frac{\lambda+\alpha+\mu-\sqrt{(\lambda+\alpha+\mu)^{2}-4 \lambda \mu}}{2 \lambda}<1$ is a simple pole of

$$
\frac{\lambda(\lambda+\alpha-\lambda \xi)}{\theta[\mu+\xi(\lambda \xi-\lambda-\alpha-\mu)]}=\frac{\lambda(\lambda+\alpha-\lambda \xi)}{\theta\left[\lambda \xi^{2}-(\lambda+\alpha+\mu) \xi+\mu\right]} .
$$

By the residue theorem, we determine

$$
\begin{aligned}
\int_{0}^{1} & \frac{\lambda(\lambda+\alpha-\lambda \xi)}{\theta[\mu+\xi(\lambda \xi-\lambda-\alpha-\mu)]} d \xi \\
= & \frac{1}{\theta} \lim _{\xi \rightarrow \frac{\lambda+\alpha+\mu-\sqrt{(\lambda+\alpha+\mu)^{2}-4 \lambda \mu}}{2 \lambda}} \frac{\lambda+\alpha-\lambda \xi}{\frac{\lambda+\alpha+\mu+\sqrt{(\lambda+\alpha+\mu)^{2}-4 \lambda \mu}}{2 \lambda}} \\
= & \frac{1}{\theta} \frac{\lambda+\alpha-\frac{\lambda+\alpha+\mu-\sqrt{(\lambda+\alpha+\mu)^{2}-4 \lambda \mu}}{2}}{\frac{\lambda+\alpha+\mu-\sqrt{(\lambda+\alpha+\mu)^{2}-4 \lambda \mu}}{2 \lambda}-\frac{\lambda+\alpha+\mu+\sqrt{(\lambda+\alpha+\mu)^{2}-4 \lambda \mu}}{2 \lambda}} \\
= & \frac{\lambda}{\theta} \frac{2(\lambda+\alpha)-\left[\lambda+\alpha+\mu-\sqrt{(\lambda+\alpha+\mu)^{2}-4 \lambda \mu}\right]}{-2 \sqrt{(\lambda+\alpha+\mu)^{2}-4 \lambda \mu}} \\
= & \frac{\lambda}{\theta} \frac{\lambda+\alpha-\mu+\sqrt{(\lambda+\alpha+\mu)^{2}-4 \lambda \mu}}{-2 \sqrt{(\lambda+\alpha+\mu)^{2}-4 \lambda \mu}} .
\end{aligned}
$$

From which together with (3.38) and (3.39) we deduce

$$
\begin{aligned}
\sum_{i=0}^{\infty} p_{I, i, 0} & =\lim _{z \rightarrow 1} Q_{1}(z) \\
& =\lim _{z \rightarrow 1} p_{I, 0,0} e^{\int_{0}^{z} \frac{\lambda(\lambda+\alpha-\lambda \xi)}{\theta[\mu+\xi(\lambda \xi-\lambda-\alpha-\mu)} d \xi} \\
& =p_{I, 0,0} e^{\int_{0}^{1} \frac{\lambda(\lambda+\alpha-\lambda \xi)}{\theta[\mu+\xi(\lambda \xi-\lambda-\alpha-\mu)]} d \xi} \\
& =p_{I, 0,0} e^{\frac{\lambda}{\theta} \frac{\lambda+\alpha-\mu+\sqrt{(\lambda+\alpha+\mu)^{2}-4 \lambda \mu}}{-2 \sqrt{(\lambda+\alpha+\mu)^{2}-4 \lambda \mu}}} \\
& <\infty
\end{aligned}
$$




$$
\begin{aligned}
\sum_{i=0}^{\infty} p_{w, i, 1}(x)= & \lim _{z \rightarrow 1} Q_{2}(x, z) \\
= & \lambda p_{I, 0,0} \lim _{z \rightarrow 1}\left\{1+\frac{\lambda+\alpha-\lambda z}{\mu+z(\lambda z-\lambda-\alpha-\mu)}\right\} \\
& \quad \times e^{\int_{0}^{1} \frac{\lambda(\lambda+\alpha-\lambda \xi)}{\overline{\theta[\mu+\xi(\lambda \xi-\lambda-\alpha-\mu)]}} d \xi} e^{-(\alpha+\mu) x} \\
= & 0 .
\end{aligned}
$$

Since Theorem 2.2 shows that each $p_{w, i, 1}(x)$ is nonnegative, (3.42) implies $p_{w, i, 1}(x)=0$ for all $i \geq 0$. This together with (3.20) and (3.21) give $p_{I, i, 0}=0$ for all $i \geq 0$. In other words, $(A+U+E)\left(p_{I}, p_{w}\right)=0$ has only the zero solution, i.e., 0 is not an eigenvalue of $A+U+E$.

Lemma 3.3 shows that the system (1.6) does not have nonzero steady-state solutions. Hence, the hypothesis 2 does not hold.

If we can prove that 0 is not in the residue spectrum of $A+U+E$, then by Theorem 2.1, Lemma 3.2, Lemma 3.3 and ABLV theorem (see [3] or [10]), we deduce that the time-dependent solution of the system (1.6) is strongly asymptotically stable. This result is quite different from other queueing models, see Gupur et al. [7]. Hence, we conclude that the results of Wang et al. [11] do not hold.

Acknowledgments The author completed this work during his stay at the Department of Mathematics and Statistics, York University.

Open Access This article is distributed under the terms of the Creative Commons Attribution Noncommercial License which permits any noncommercial use, distribution, and reproduction in any medium, provided the original author(s) and source are credited.

\section{References}

1. Adams, R.A.: Sobolev Space. Academic Press, New York (1975)

2. Aleksandrov, A.M.: A queueing system with repeated orders. Eng. Cybernet. Rev. 12(3), 1-4 (1974)

3. Arendt, W., Batty, C.J.K.: Tauberian theorems and stability of one-parameter semigroups. Trans. Am. Math. Soc. 306, 837-852 (1988)

4. Choi, B.D., Park, K.K.: The M/G/1 retial queue for Bernoulli schedule. Queueing Syst. 7, 219228 (1990)

5. Falin, G.I.: On the waiting-time process in a single-line queue with repeated calls. J. Appl. Probab. 23, 185-192 (1986)

6. Gupur, G.: Semigroup method for M/G/1 retrial queue with general retrial times. Int. J. Pure Appl. Math. 18, 405-429 (2005)

7. Gupur, G., Li, X., Zhu, G.: Functional Analysis Method in Queueing Theory. Research Information Ltd, Hertfordshire (2001)

8. Keilson, J.C., Young, H.: A service system with unfilled requests repeated. Oper. Res. 16, 11261137 (1968)

9. Kulkarni, V.G.: On queueing systems with retrials. J. Appl. Probab. 20, 380-389 (1983)

10. Lyubich, Y.I., Phong, V.Q.: Asymptotic stability of linear differential equations in Banach spaces. Studia Math. 88, 37-42 (1988)

11. Wang, J., Cao, J., Li, Q.: Reliability analysis of the retrial queue with server breakdowns and repairs. Queueing Syst. 38, 363-380 (2001)

12. Yang, T., Templeton, J.G.C.: A survey on retrial queues. Queueing Syst. 2, 201-233 (1987) 